\title{
COMPETITIVE ACCRETION AND THE IMF
}

\author{
Ian A. Bonnell \\ School of Physics and Astronomy, University of St Andrews, North Haugh, St Andrews, \\ KY16 9SS, UK \\ iab1@st-and.ac.uk
}

\begin{abstract}
Competitive accretion occurs when stars in a cluster accrete from a shared reservoir of gas. The competition arises due to the relative attraction of stars as a function of their mass and location in the cluster. The low relative motions of the stars and gas in young, gas dominated clusters results in a tidal limit to the accretion whereas in the stellar dominated cluster cores, the high relative velocities results in Bondi-Hoyle accretion. The combination of these two accretion processes produces a two power-law IMF with $\gamma \approx-1.5$, for low-mass stars which accrue their mass in the gas dominated regime, and a steeper, $\gamma \approx-2.5$, IMF for higher-mass stars that form in the core of a cluster. Simulations of the fragmentation and formation of a stellar cluster show that the final stellar masses, and IMF, are due to competitive accretion. Competitive accretion also naturally results in a mass segregated cluster and in a direct correlation between the richness of a cluster and the mass of the most massive star therein. The knee where the IMF slope changes occurs near the Jeans mass of the system.
\end{abstract}

\section{Introduction}

Star formation is a dynamical process where most stars form in a clustered ennvironment (Lada \& Lada 2003; Clarke, Bonnell \& Hillenbrand 2000). In such an environment, stars and gas move in their combined potential on timescales comparable to the formation time of individual stars. Furthermore, the initial fragmentation of a molecular cloud is very inefficient (eg., Motte et al. 1998), such that the youngest clusters are dominated by their gas content. In such an environment, gas accretion can contribute significantly to the final mass of a star.

Stellar clusters are found to be mass segregated even from the youngest ages. The location of massive stars in the cores of clusters cannot be explained by dynamical mass segregation as the systems are too young 
(Bonnell \& Davies 1998). A Jeans mass argument also fails as the Jeans mass in the core should be lower then elsewhere in the cluster. Mass segregation is a natural outcome of competitive accretion due to the gas inflow to the centre of the cluster potential.

\section{Accretion in stellar clusters: two regimes}

In a series of numerical experiments, we investigated the dynamics of accretion in gas-dominated stellar clusters (Bonnell et al. 1997, 2001a). In the initial studies of accretion in small stellar clusters, we found that the gas accretion was highly non-uniform with a few stars accreting significantly more than the rest. This occurred as the gas flowed down to the core of the cluster and was there accreted by the increasingly mostmassive star. Other, less massive stars were ejected from the cluster and had their accretion halted (see also Bate, Bonnell \& Bromm 2002). In a follow-up study investigating accretion in clusters of 100 stars, we discovered two different physical regimes (Bonnell et al. 2001a) resulting in different accretion radii, $R_{\text {acc }}$, for the mass accretion rate

$$
M_{\mathrm{acc}}=\rho v \pi R_{\mathrm{acc}}^{2},
$$

where $\rho$ is the local gas density and $v$ is the relative velocity of the gas. Firstly, in the gas dominated phase of the cluster, tides limit the accretion as the relative velocity between the stars and gas is low. The tidal radius, due to the star's position in cluster potential,

$$
R_{\text {tidal }} \approx 0.5\left(\frac{M_{*}}{M_{\text {enc }}}\right)^{\frac{1}{3}} R_{*},
$$

is then smaller than the traditional Bondi-Hoyle radius and determines the accretion rate. Accretion in this regime naturally results in a mass segregated cluster as the accretion rates are highest in the cluster core.

Once accretion has increased the mass of the stars, and consequently decreased the gas mass present, the stars begin to dominate the stellar potential and thus virialise. This occurs first in the core of the cluster where higher-mass stars form due to the higher accretion rates there. The relative velocity between the gas and stars is then large and thus the Bondi-Hoyle radius,

$$
R_{\mathrm{BH}}=2 G M_{*} /\left(v^{2}+c_{s}^{2}\right),
$$

becomes smaller than the tidal radius and determines the accretion rates.

\section{Resultant IMFs}

We can use the above formulation of the accretion rates, with a simple model for the stellar cluster in the two physical regimes, in order to 
derive the resultant mass functions (Bonnell et al. 2001b). The primary difference is the power of the stellar mass in the accretion rate equation, $M_{*}^{2 / 3}$ for tidal-accretion and $M_{*}^{2}$ for Bondi-Hoyle accretion. Starting from a gas rich cluster with equal stellar masses, tidal accretion results in higher accretion rates in the centre of the cluster where the gas density is highest. This results in a spread of stellar mass and a mass segregated cluster. The lower dependency of the accretion rate on the stellar mass results in a fairly shallow IMF of the form (where Salpeter is $\gamma=-2.35$ )

$$
d N / d M_{*} \propto M_{*}^{-3 / 2} .
$$

Once the cluster core enters the stellar dominated regime where BondiHoyle accretion occurs, the higher dependency of the accretion rate on the stellar mass results in a steeper mass spectrum. Zinnecker (1982) first showed how a Bondi-Hoyle type accretion results in a $\gamma=-2$ IMF. In a more developed model of accretion into the core of a cluster with a pre-existing mass segregation, the resultant IMF is of the form

$$
d N / d M_{*} \propto M_{*}^{-5 / 2} .
$$

This steeper IMF applies only to those stars that accrete the bulk of their mass in the stellar dominated regime, ie. the high-mass stars in the core of the cluster (see Figure 1).

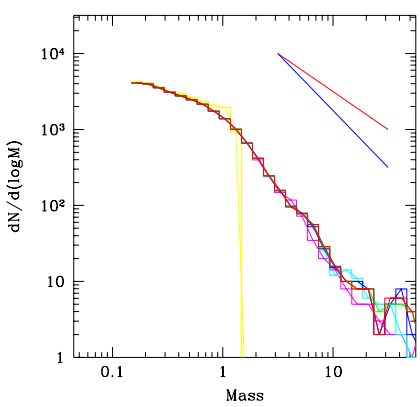

Figure 1. The IMF that results from competitive accretion in a model cluster. the two power-law IMF results from a combination of tidal accretion for low-mass stars and BondiHoyle accretion for high-mass stars (BCBP 2001).

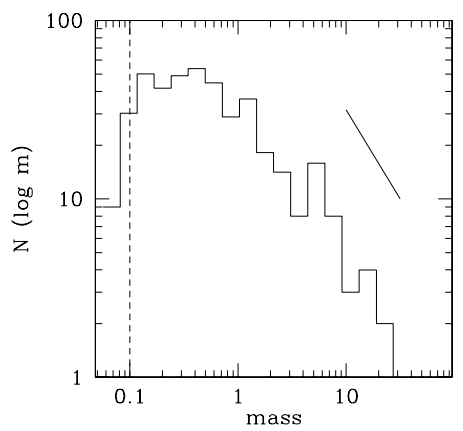

Figure 2. The IMF that results from a numerical simulation of the ffragmentation of a turbuelnt cloud and the formation of a stellar cluster containing 419 stars. The comparison slope is for a $\gamma=-2$ IMF (BBV 2003). 


\section{The formation of stellar clusters}

In order to asses the role of accretion in determining the IMF, we need to consider self-consistent models for the formation of the cluster. We followed the fragmentation of a $1000 \mathrm{M}_{\odot}$ cloud with a $0.5 \mathrm{pc}$ radius (Bonnell, Bate \& Vine 2003). The cloud is initially supported by turbulence which decays on the cloud's crossing time. As the turbulence decays, it generates filamentary structure which act as the seeds for the subsequent fragmentation. The cluster forms in a hierarchical manner with many subclusters forming before eventually merging into one larger cluster. In all 419 stars form in $5 \times 10^{5}$ years. The simulation produces a field star IMF with a shallow slope for low-mass stars steepening to a Salpeter-like slope for high-mass stars (Figure 2). The stars all form with low masses and accrete up to their final masses. The stars that are in the centres of the subclusters accrete more gas and thus become higher-mass stars.

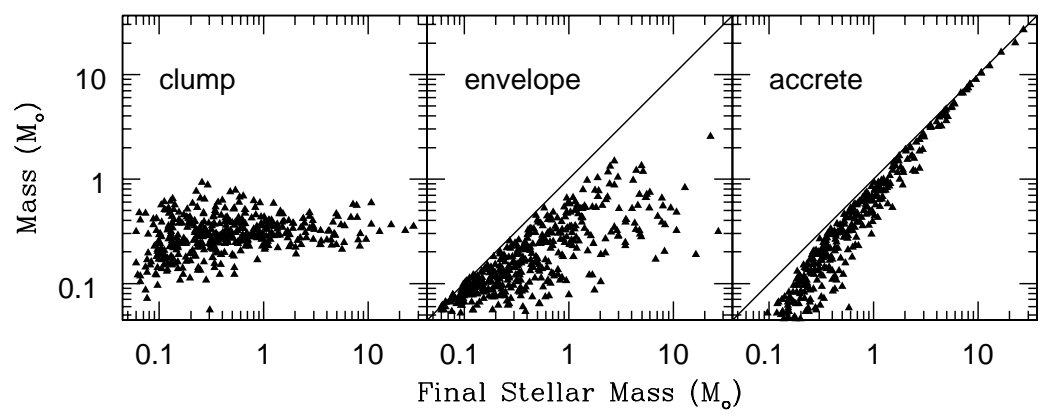

Figure 3. The relation between fragment mass (left) mass in a contiguous envelope (middle) and mass accreted from beyond the cluster (right) is plotted against final stellar mass. High-mass stars attain their mass due to competitive accretion of gas infalling into the stellar cluster (Bonnell, Vine \& Bate 2004).

A careful dissection of the origin of the more massive stars reveals the importance of competitive accretion in setting the IMF. the Lagrangian nature of the SPH simulations allows us to trace the mass from which a star forms. We can therefore distribute this mass as being in one of three categories: 1) The original fragment which formed the star; 2) A contiguous envelope around the fragment until we reach the next forming star; and 3) mass which originates beyond the forming group or cluster of stars. Figure 3 shows the three contributions to the final stellar mass of the 419 stars (Bonnell, Vine \& Bate 2004). We see that the initial fragment mass is generally around $0.5 M_{\odot}$ and fails to account for both 
high-mass and low-mass stars. The contiguous envelope does a better job as it accounts for subfragmention into lower-mass stars. It still fails to explain the mass of higher-mass stars. We are thus left with accretion from beyond the forming protocluster in order to explain the existence of higher-mass stars. Thus, high-mass stars are formed due to competive accretion of gas that infalls into the stellar cluster.

\section{Massive stars and cluster formation}

One of the predictions that we can extract from the simulations is that there should be a strong correlation between the mass of the most massive star and the number of stars in the cluster (Figure 4, Bonnell, Vine \& Bate 2004). This occurs due to the simultaneous accretion of stars and gas into a forming stellar cluster. Given an effective initial efficiency of fragmentation, for every star that falls into the cluster a certain amount of gas also enters the cluster. This gas joins the common reservoir from which the most massive star takes the largest share in this competitive environment. Thus, the mass of the most massive star increases as the cluster grows in numbers of stars.

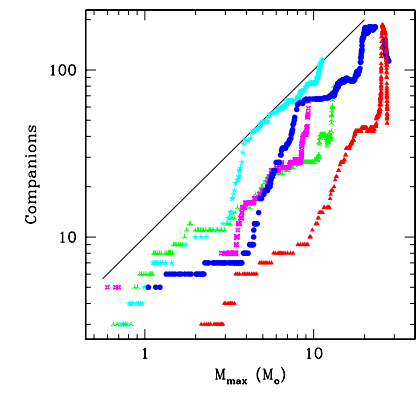

Figure 4. The number of stars in a subcluster is plotted against the mass of the most massive star therein(BVB 2004).

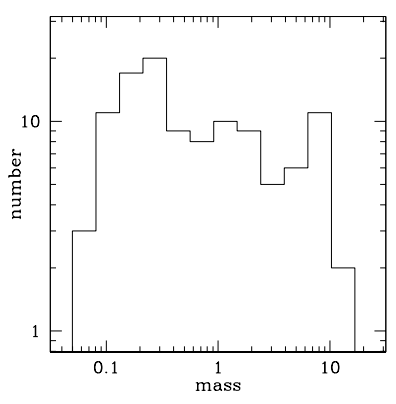

Figure 5. The IMF that results from a fragmentation of a $1000 \mathrm{M}_{\odot}$ cloud where the Jeans mass is $5 \mathrm{M}_{\odot}$..

\section{What is the role of the Jeans mass?}

If competitive accretion is the determining factor in setting the IMF, what role does the Jeans mass play? We saw above that the average fragment mass in which stars form is $\approx 0.5 \mathrm{M}_{\odot}$ while the initial Jeans mass is $=1 M_{\odot}$. Thie Jeans mass is therefore important at setting the scale of the initial fragmentation. We have run a simulation identical to 
the one in Bonnell, Bate \& Vine (2003) except with a Jeans mass of $5 M_{\odot}$. The resultant IMF, shown in Figure 5, is fairly shallow up to masses of $\approx 5-10 M_{\odot}$, corresponding roughly to the Jeans mass. In Figure 2 we see that the slope of the IMF becomes steeper at $\approx 1 M_{\odot}$, the Jeans mass in this simulation. We can thus deduce that the Jeans mass helps set the knee in the IMF, with lower masses determined by fragmentation and the tidal shearing of nearby stars, while higher-masses are determined by accretion in a clustered environment.

\section{Conclusions}

Competiitve accretion is a simple physical model that can explain the origin of the initial mass function. It relies on gravitational competition for gas in a clustered environment and does not necessarily involve large-scale motions of the accretors. The two physical regimes (gas and stellar dominated potentials) naturally result in a two power-law IMF. It is probably even more important for any model of the IMF to have secondary characteristics that can be compared to observations. For example, competive accretion naturally results in a mass segregated cluster, and predicts that massive star formation is intrinsically linked to the formation of a stellar cluster. Some of the simulations reported here were performed with the UK's Astrophysical Fluid facility, UKAFF.

\section{References}

Bate, M. R., Bonnell, I. A., Bromm, V. 2002, MNRAS, 336, 705

Bonnell, I. A., Bate, M. R., Clarke, C. J., \& Pringle, J. E., 1997, MNRAS, 285, 201

Bonnell, I. A., Bate, M. R., Clarke, C. J., \& Pringle, J. E., 2001, MNRAS, 323, 785

Bonnell, I. A., Bate, M. R., \& Vine, S. G. 2003, MNRAS, 343,413

Bonnell, I. A., Clarke, C. J., Bate, M. R., \& Pringle, J. E., 1997, MNRAS, 324, 573

Bonnell, I. A., Davies, M. B., 1998, MNRAS, 295, 691

Bonnell, I. A., Vine, S. G., \& Bate, M. R. 2004, MNRAS, 349, 735

Clarke, C. J., Bonnell, I. A., Hillenbrand, L. A., 2000, PPIV, Mannings, Boss \& Russell eds, p 151

Lada C. J., Lada, E. 2003, ARA\&A,

Motte, F., Andre, P., \& Neri, R. 1998, A\&A, 336, 150

Salpeter, E. E. 1955, ApJ, 123, 666

Zinnecker, H. 1982, New York Academy Sciences Annals, 395, 226 